\title{
Cochlear blood flow measured by averaged laser Doppler flowmetry (ALDF)
}

\author{
Tianying Ren ${ }^{a, b}$, P. Bradley Brechtelsbauer a , Josef M. Miller a , Alfred L. Nuttall a,* \\ "Kresge Hearing Research Institute, The Unirersity of Michigan Medical School, 1301 East Ann Street, Ann Arbor, MI 48109-0506, USA \\ "Department of Onlaryngology, Xian Medical Linicersity, Xian, China
}

(Received 24 October 1993; Revision received 4 March 1994; Accepted 13 March 1994)

\begin{abstract}
This report describes a new approach to estimate the hydromechanical propertics of a vascular system. Averaged laser Doppler flowmetry (ALDF) was developed by averaging the flux signal of a laser Doppler flowmeter (LDF) synchronized to the heart cycle. The usefulness of this method was verified by manipulation of the cochlear microvasculature. Twelve pigmented guinea pigs under pentobarbital/fentanyl anesthesia were used. The cochlea was surgically exposed and the LDF probe placed on the bony surface of the first turn to monitor cochlear blood flow (CBF). The LDF flux signal (0.2 s time constant) was sampled by an A/D board at $2 \mathrm{kHz}$ for $255 \mathrm{~ms}$ and averaged with synchronization to the heart beat. The mean blood flow, peak to peak amplitude, and time (phase) delay of pulsatile flow were measured from the averaged signal. According to a transmission line model of the vascular system, under a given perfusion pressure, mean flow reflects resistance while amplitude and time delay of the pulsatile flow are related to the reactance component of the impedance of the vascular system. During the formation of photochemically-induced thrombosis in the cochlear microvasculature, there was a dramatic mean flux decrease (90.1 $\pm 3.4 \%$ from baseline (BL), $N=6$ ). Additionally, a time-dependent decrease in amplitude and time delay of pulsatile flow were indicated by ALDF. These results suggest a large increase in vascular resistance and significant decrease in compliance. After application of $2 \%$ sodium nitroprusside (NP) to the round window membrane, mean flux increased by $78.3 \pm 17.6 \%$ BL: amplitude and time delay of pulsate blood flow increased by $81.0 \pm 14.6 \%(N=6)$ and $11.5 \pm 3.2 \mathrm{~ms}(N=6)$, respectively. These changes can be interpreted as lowered resistance and increased compliance consistent with vasodilatation by NP. ALDF is a new method which derives additional information from the LDF signal. The new information provided by ALDF can be of importance in assessment of basic cochlear microcirculation and interpretation of peripheral vascular diseases in the cochlea and other organs.
\end{abstract}

Key words: Microcirculation; Cochlear blood flow; Laser Doppler flowmetry; Signal averaging; Pulse blood flow; Pulse blood pressure

\section{Introduction}

Laser Doppler flowmetry (LDF) has been extensively used in microvascular research and in the clinical asscssment of tissue perfusion for the past decade. The technique has many attractive features including (1) ease of operation, (2) continuous measurement, and (3) noninterference with blood flow in the microcirculation, since the technique involves illuminating the tissue with monochromatic light. These attributes of LDF have led several research groups to utilize the tech-

\footnotetext{
* Corresponding author. Fax: (313) 764-0014.
}

nique in the assessment of tissue perfusion in various vascular beds, including skin (Johnson, 1984), the gastrointestinal system (Shepherd and Redel, 1982), skeletal muscle (Tyml et al., 1990), kidney (Roman and Smits, 1985), brain (Haberl et al., 1989), bone (Hellem et al., 1983), nose (Druce et al., 1984), eye (Riva et al., 1990), and cochlea (Miller and Nuttall, 1990).

Without doubt, a major technical advance in cochlear blood flow (CBF) study nccurred within the last decades with the introduction of the laser-Doppler flowmeter and its application in studies of CBF (Miller et al., 1983). Since LDF can offer dynamic measurement of CBF, it has been used in studies of vasoactive agents (Quirk et al., 1988; Ohlsèn et al., 1991; Laurikainen et al., 1993a), local electrical stimulation (Sill- 
man et al., 1989), neural control (Ren et al., 1991; Laurikainen et al., 1993b; Ren et al, 1993a,b), and vasomotion (Ren et al., 1993c) in guinea pig cochlea. LDF differs from electrical impedance measurement (Suga and Snow, 1969) since it can measure both steady and pulsatile flow, while the electrical impedance technique responds only to pulsatile flow. However, routine LDF flux recordings show only steady blood flow and provide little information about pulsatile blood flow because of the low signal to noise ratio. According to the theories of hemodynamics, the transmissions of pulse pressure and pulsatile flow are determined by the vascular mechanical properties, such as resistance and compliance, which play a critical role in vascular disease. The purpose of this study was to develop the technique of averaged laser Doppler flowmetry (ALDF) in order to estimate changes in vascular mechanical properties, and to verify its usefulness by manipulation of the cochlear microvasculature.

\section{Methods}

\section{Animals and surgical approach}

Twelve healthy young guinea pigs weighing $250-420$ $\mathrm{g}$ were used in this study. After light sedation with pentobarbital $(15 \mathrm{mg} / \mathrm{kg} \mathrm{IP})$, analgesia was provided by fentanyl $(0.32 \mathrm{mg} / \mathrm{kg}$ IM). Anesthesia was maintained by a supplementary half dose of fentanyl every $30 \mathrm{~min}$ and half dose of pentobarbital every $2 \mathrm{~h}$. A ventilation tube was inserted into the trachea to insure free breathing. Rectal temperature was maintained at $38 \pm 1^{\circ} \mathrm{C}$ with a servo-regulated heating blanket.

The left or right carotid artery was cannulated and hydraulically connected to a pressure transducer (P23KL, Statham Instruments, Inc., Oxnard, CA) for continuous blood pressure (BP) measurement with a custom-made electrical circuit. Two stainless steel needle electrodes were placed subcutaneously on the anterior chest wall and a custom-made amplifier was used for electrocardiogram (ECG) recording. Animals with mean BP above $40 \mathrm{mmHg}$ were used for data collection. While not assessed in the subjects of this study, from extensive laboratory experience with this anesthesia in the guinea pig, animals with these blood pressures routinely demonstrate blood gas values within a normal range.

This study was performed in accordance with the PHS Policy on Humane Care and Use of Laboratory Animals; the animal use protocol was approved by the Animal Care and Use Committee of the University of Michigan.

\section{Laser Doppler flowmetry and signal averaging}

For LDF, the bulla was exposed using a ventral approach, and the cochlear mucosa was gently re- moved with a small cotton pledget. The laser probe (PF 303, Perimed Co., Stockholm, Sweden) was placed on the lateral wall of the first turn of the cochlea. CBF was monitored with a Periflux PF2B laser Doppler flowmeter (Perimed Co., Stockholm, Sweden). The time constant was set to $0.2 \mathrm{~s}$ and the frequency cutoff to 12 $\mathrm{kHZ}$.

ECG, BP, and CBF signals were recorded with a Racal Store 4DS FM recorder (Racal Recorders Ltd., England). When the signals were played back, the following off-line analysis was performed, using a computerized averaging system. The flux signal from the LDF was averaged 256 times, synchronized with heart beat. The triggering signal was generated by a custommade power switching driver circuit, using FCG as the input signal of the operational amplifier. Analog to digital sample frequency was $2 \mathrm{kHz}$, and the signal window ( $255 \mathrm{~ms}$ width) consisted of 510 data points. To obtain phase information, the time delay from the start of triggering signal to the first trough of the averaged CBF wave was measured on screen. The peak to peak and the mean amplitudes were also acquired to estimate the pulsatile and steady CBF.

The pulse BP signal also was averaged with the same technique, and the time delay from triggering signal to the first trough was obtained. Because of considerable variation in systolic and diastolic BP values over time, the systolic to diastolic amplitude were not used in the current study.

\section{Photochemically-induced thrombosis in the cochlea}

Rose bengal (Sigma, USA) was infused intravenously via the external jugular vein at a rate of 1 $\mathrm{ml} / \mathrm{kg} / \mathrm{h}(24 \mathrm{mM})$, as reported by Umemura et al. (1990). Photo-illumination of the lateral wall of the cochlea was started $30 \mathrm{~min}$ after the initiation of the continuous infusion. Light was supplied to a 5 foot long, $3 \mathrm{~mm}$ diameter, liquid light-guide from a $100 \mathrm{~W}$ xenon lamp. A heat-absorbing filter and a high transmission bandpass filter $(470-570 \mathrm{~nm})$ were placed between the light source and the light-guide, and the end of the liquid light-guide was place approximately $7 \mathrm{~mm}$ away from the lateral wall of the cochlea. The illuminated was centered over the area on the basal turn contacted with the laser probe. The rose bengal infusion was continued during the illumination.

\section{Topical application of agents on the round window mem brane}

Followed the procedure reported by Ohlsèn et al. (1991), $20 \mathrm{mg}$ sodium nitroprusside (NP) (Sigma, USA) in $1 \mathrm{ml}$ sterile water was prepared and $1 \mu l$ of this solution was applied on the round window membrane. $\mathrm{CBF}$ and $\mathrm{BP}$ responses were monitored. 


\section{Experimental protocol}

Six animals were used to test the effects of microvascular thrombosis on the averaged LDF signal. The baseline measurement of ALDF was taken before illumination, and sequential measurements were taken $2,4,6,10$, and 15 min after the initiation of illumination. The effect of topical NP on ALDF measurement was observed in another six animals. Baseline measurement was taken immediately before the application of NP to the round window membrane. Five min after NP application sequential measurement was initiated.

\section{Statistical analysis}

ALDF values obtained before the manipulation represented the baseline measurement. Values presented are means \pm standard deviations unless otherwise stated. Nonlinear regressions were used to reveal the relationships among the different parameters. A least squares method was used to select equation parameters to fit an equation to data. Regression line was generated from the equation. Pearson product moment correlation analysis was used to calculate correlation coefficient.

\section{Results}

\section{Averaged laser Doppler flowmetry (ALDF)}

In Fig. 1, the relationships of the ECG, triggering signal, pulse BP, and CBF are shown. Depolarization waves of cardiac ventricles, the QRS wave complex can be clearly identified. Although the configuration and amplitude of the QRS complex vary considerably among individuals, the duration is usually $12-20 \mathrm{~ms}$ and has one or two sharp peaks. The QRS complex in Fig. 1 consists of $R$ and $S$ waves with almost the same amplitude without a visible $Q$ wave. When the potential level reaches the threshold between $P, T$, waves, and the positive peak of QRS waves, indicated by line $\mathrm{O}$, the triggering signal is generated. The width of the triggering signal was determined by the width of the QRS complex at threshold level; the amplitude of the trigger signal was 3 volts in this experiment. Based on the heart beat period R-R or S-S, the heart rate can be accurately calculated. The heart rate in 12 animals was $346.7 \pm 18.5$ cycle per min. In addition, the shape of the ECG can provide information on heart function.

The avcraged CBF in Fig. 1 shows a very clear oscillation with the same frequency as $\mathrm{HR}$ and a phase different from ECG and BP. Moreover, the shape of averaged CBF is different from that of BP, presenting an almost perfect sinusoidal curve. The peak to peak amplitude of flux signal in percentage of the mean value (vertical distance between $\mathbf{N}$ and $\mathbf{M}$ ) describes a heart beat-related pulsate flow. The peak to peak amplitude of pulsatile flow was $1.63 \pm 0.32 \%$ of the
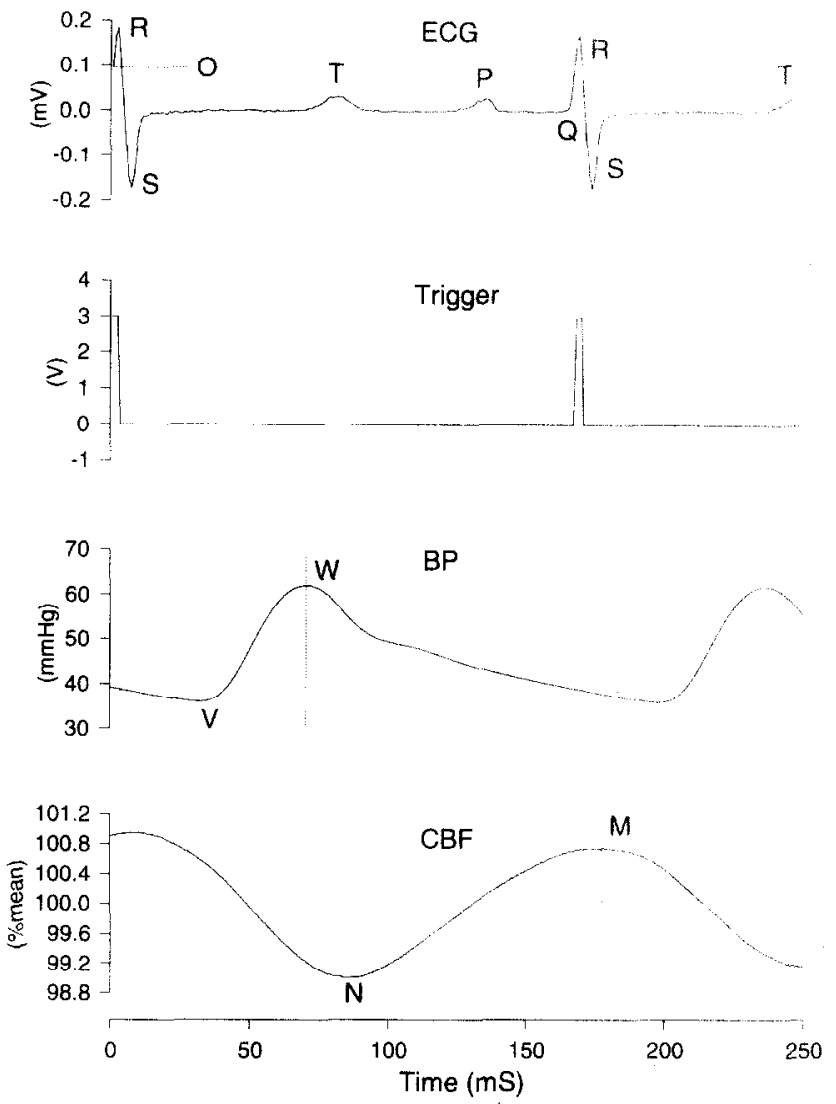

Fig. 1. A typical averaged CBF and its relationship to ECG, trigger, BP. ECG of one heart beat cycle consists of $P, R, S, T$ waves in the ECG panel. The dashed line $O$ indicates the threshold for the power switch driver to generate the triggering signal. The trigger panel shows two positive square pulses with a 3 volt amplitude. The second pulse of the trigger signal cannot activate the averaging system since the width of the signal window was fixed at $255 \mathrm{~ms}$. In the BP panel, the horizontal distance from zero to $W$ and the vertical distance between $W$ and $V$ indicate time delay and amplitude of pulsatile pressure, respectively. In the CBF panel, the horizontal distance from zero to $M$ and the vertical distance between $M$ and $N$ indicate the the time delay and amplitude of pulsatile flow.

mean $\mathrm{CBF}$ value $(N=12)$ before manipulation of the cochlear microvasculature. The time delay from the start of the triggering signal to the trough of the averaged CBF varied among the animals. Amplitude of pulse pressure, the pressure difference between systolic pressure (W) and diastolic pressure (V), changed over time due to the unstable saline-blood interface in the catheter. As for averaged CBF, pulsatile pressure also showed various time delays.

Averaged laser Doppler flowmetry (ALDF) changes caused by photochemically-induced thrombosis in the cochlea

In Fig. 2, CBF during the formation of cochlear thrombosis is plotted against the exposure time; $100 \%$ $\mathrm{BL}$ is the mean value of averaged $\mathrm{CBF}$ immediately before illumination. The dramatic $\mathrm{CBF}$ reduction, from 


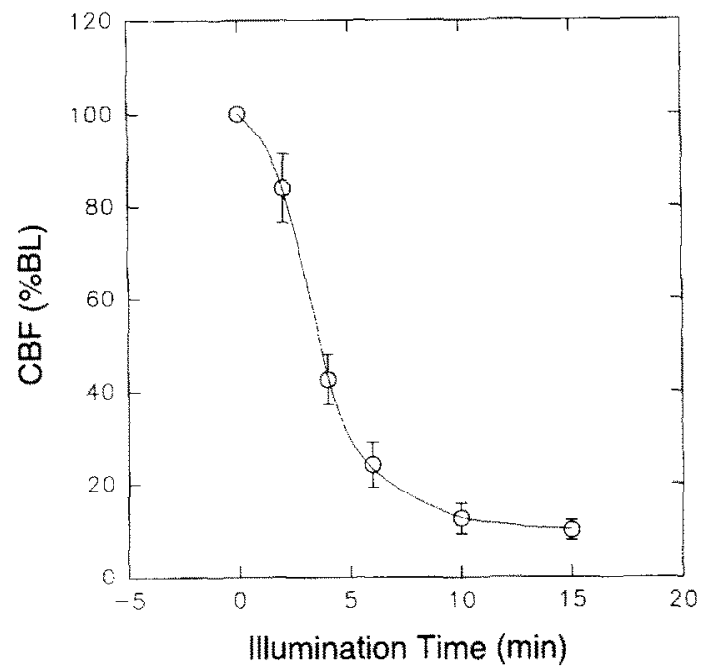

Fig. 2. The change in steady flow over the illumination period is shown. The dramatic CBF reduction, from $100 \% \mathrm{BL}$ to about $20 \%$ $\mathrm{BL}$, occurred during the first 5 min of illumination; then $\mathrm{CBF}$ tended to remain at about $9 \% \mathrm{BL}$. Correlation analysis $(\mathrm{r}=-0.880, P=$ $0.021, N=6$ ) suggests a negative nonlinear relationship between exposure time and $\mathrm{CBF}$.

$100 \% \mathrm{BL}$ to about $20 \% \mathrm{BL}$, occurred during the first 5 min illumination; thereafter, $\mathrm{CBF}$ tended toward an asymptote at approximately $9 \% \mathrm{BL}$. CBF never reached biological zero, which is the laser Doppler output after death. Correlation coefficient $(\mathrm{r}=-0.880, P=0.021$, $N=6$ ) suggests a strong negative nonlinear relationship between the exposure time and steady CBF.

Pulse flow intensity is graphed against illumination time in Fig. 3. The normalized pulse flow was used to estimate the intensity of pulse flow and is presented as

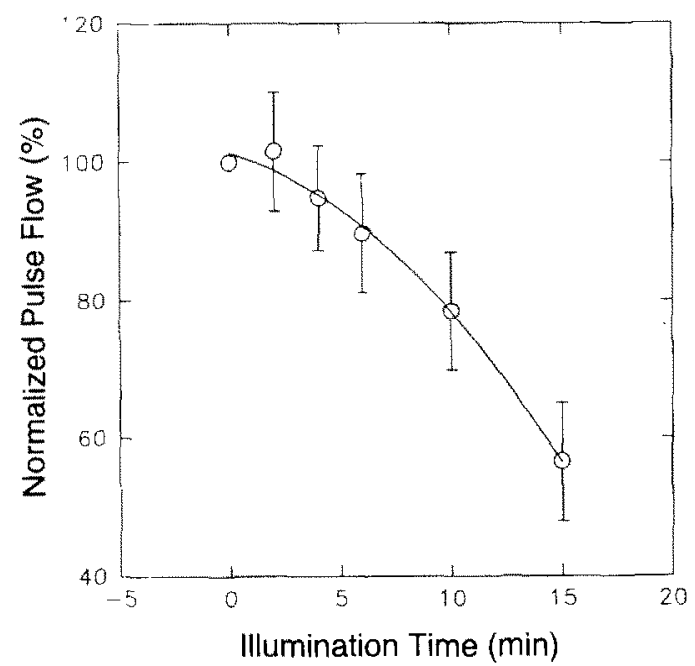

Fig. 3. A very strong nonlinear negative relationship is shown between pulse flow intensity and illumination time $(\mathrm{r}=-0.943, P<$ $0.001, N=6$ ). Normalized pulse flow is tefined as the percentage of pulse flow at time zero of illumination.

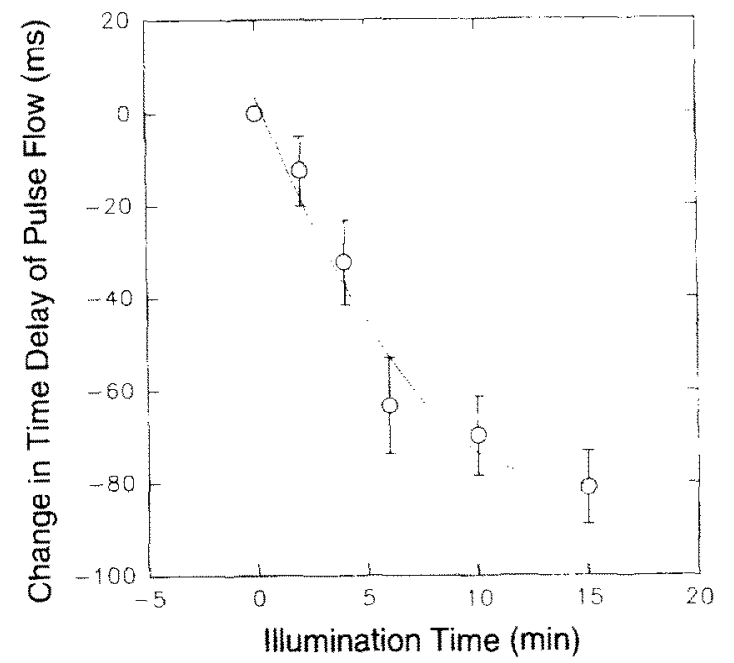

Fig. 4. A strong nonlinear negative relationship was found between time delay of pulse flow and illumination time $(r=-0.930, P=0.007$. $N=6$ ).

the percent of pulse flow in arbitrary units at time zero of illumination. The pulse flow intensity tended to increase at $2 \mathrm{~min}$ of illumination and then decreased. The decreased amplitude of pulse flow, approximately $42 \%$ of original value, is much smaller than mean value reduction (about $90 \%$ BL in Fig. 2). A strong nonlinear negative relationship between pulse flow intensity and illumination time is suggested by correlation coefficient $(\mathrm{r}=-0.943, P<0.001, N=6)$.

Phase change of pulsatile flow over 15 min illumination is presented in Fig. 4. Phase change is defined as change in time delay of pulse flow (ms) which is the time differenee in delay at time zero of illumination to other measurements. Time delays of pulse flow gradually decreased with light exposure and tend to be saturated during the last $5 \mathrm{~min}$. Correlation analysis ( $\mathrm{r}=-0.930, P=0.007, N=6)$ indicates a strong nonlinear negative correlation between phase shift of pulsatile flow and illumination time.

The relationship of mean CBF with intensity and time delay of pulse flow were shown in Fig. 5. The solid line suggests a nonlinear negative relationship between mean flow and pulse flow. However, the correlation coefficient $(\mathrm{r}=-0.786)$ and $\mathrm{p}$ value $(P=$ 0.064 ) indicated no significant correlation between these two values. The dashed line shows the relation. ship between mean flow and the change in time delay of pulse flow. Pearson product moment correlation coefficient ( $\mathrm{r}=-0.976, P<0.001, N=6$ ) suggests a strong nonlinear negative relationship between mean blood flow and pulse flow.

Assessment of nitroprusside effect on $C B F$

After local application of NP on the round window membrane, systemic BP did not show significant 


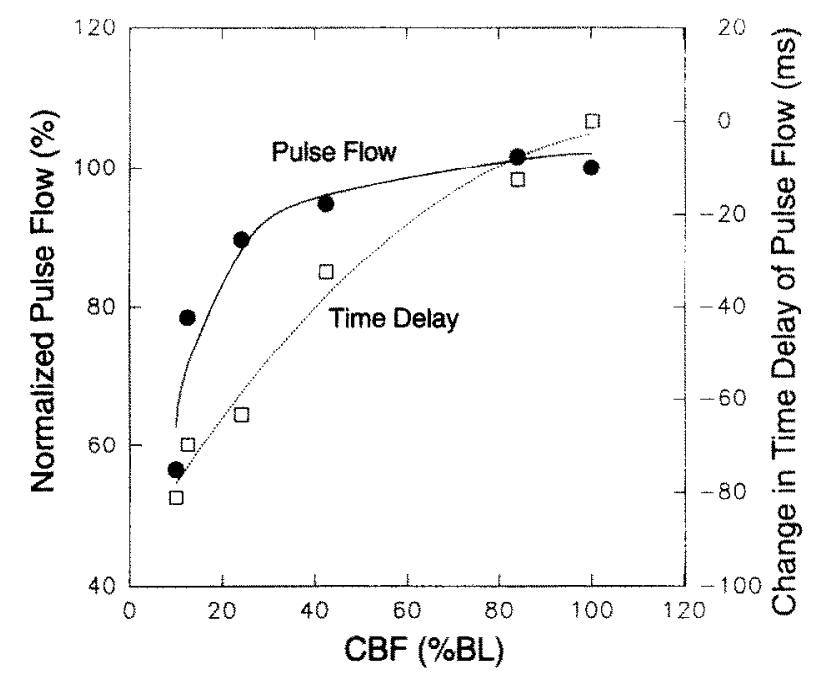

Fig. 5. The relationship of steady CBF with intensity and time delay of pulse flow is shown. The solid line suggests no significant relationship between steady flow and pulse flow $(\mathrm{r}=-0.786, P=0.064)$. The dashed line indicates a strong nonlinear negative relationship between mean blood flow and pulse flow $(\mathrm{r}=-0.976, P<0.001$, $N=6$ ).

change. CBF started to increase in about one min and reached a maximum in about five min. Recovery of $\mathrm{NP}$-provoked increase did not occur during the $30 \mathrm{~min}$ observation period. Changes in mean CBF, pulse CBF, and time delay of pulsatile flow were presented in Fig. 6. Compared with original measurement immediately before NP application, mean CBF increased by $78.3 \pm$ $17.6 \%$ BL $(N=6)$. The intensity and time delay of pulse flow increased by $81 \pm 14.6 \%(N=6)$ and $11.5 \pm$ $3.2 \mathrm{~ms}(N=6)$. These data indicate that after local NP application, both steady and pulse blood flux increased and the velocity of transmission of pulse blood flow appears slower.

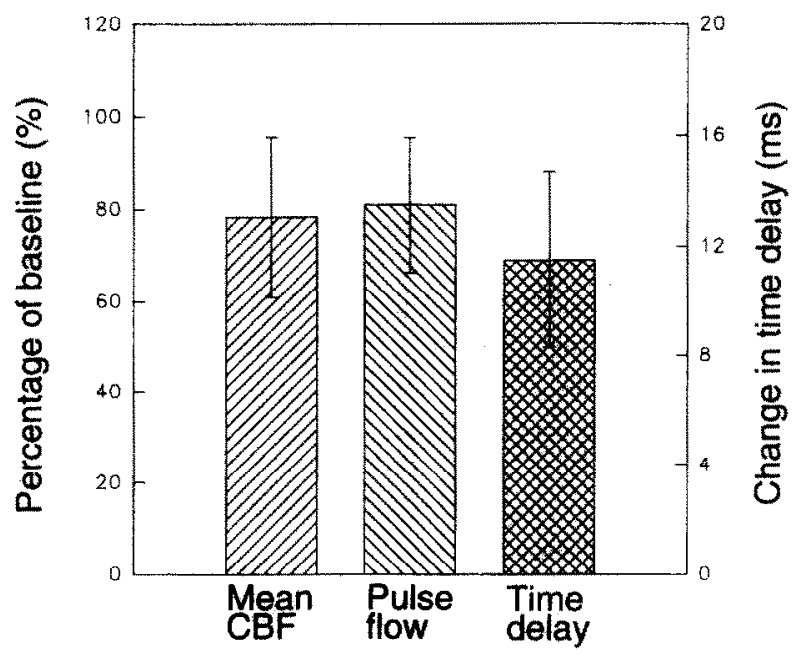

Fig. 6. Changes in mean CBF, pulsatile CBF, and time delay of pulsatile flow $5 \mathrm{~min}$ after topical NP application.

\section{Discussion}

\section{General considerations}

The relationship between pressure and flow in a small rigid tube is described by the Hagen-Poiseuille law. It states that $\mathrm{Q}=\Delta \mathrm{P} \pi \mathrm{r}^{4} / 8 \mathrm{~L} \eta$, where $\mathrm{Q}$ is the volume flow per unit time, DP is the pressure, $r$ is the radius, $L$ is the length of the tube, and $\eta$ is the viscosity of the fluid. Although this relationship holds only for the steady streamlined flow of a Newtonian fluid, its application to the circulation permits a qualitative understanding of blood flow. However, the relationship between pressure and flow in the vascular segment of the circulatory system is more complicated than described by the Hagen-Poiseuille law alone. The pulsatile nature of arterial pressure and flow combined with the elastic properties of the vessels, the viscosity and inertial nature of blood, and the variable compliance and geometry of the vascular system introduce phase relationships between pressure and flow.

Most model analyses treat blood vessels as a string of identical units, chained together so that pressure and flow waves pass through each one in sequence. The mathematical expression of such an arrangement is called a transmission-line model (Mcllroy et al., 1986; Taylor et al., 1959; Womersley, 1957). Taylor (1959) designed an electrical network for a mathematical transmission-line model to simulate the effects of viscoelasticity in the walls of blood vessels. In our previous study, an electrical analog model was developed to describe the nonlinear pressure-flow relationship of blood circulation in the guinea pig cochlea (Ren et al., 1993d).

In a sinusoidally oscillating system containing both capacitance and resistance, the peak pressure and flow are separated (the phase angle) because of the alternating storage and discharge of the capacitance. Mathematically, these factors can be combined into a single vector, the impedance. Impedance expresses the opposition offered by vessels or vascular beds to pulsatile flow, just as resistance expresses the opposition to steady flow. Both must be overcome by energy that the heart supplies to move blood in a pulsatile fashion.

Therefore, we assume that capacitance and/or resistance changes due to any physiological or pathological condition will affect vascular impedance, and hence will result in changes in pulsatile flow amplitude and in the phase relationship between peak flow and peak pressure. Thus, measurement of amplitude and phase of blood flow pulsation should reveal fundamental mechanical properties of a vascular system. With pathology, we expect these properties to be modified. Then, their specific assessment can lead to a better understanding of the changes that underlie pathologically induced alteration in blood flow. 
The interpretation of $A L D F$ measurement

Theoretically, ALDF is a pulse flow measurement of vascular mechanical status, or impedance. In the current experiment, the triggering pulse was initiated by the QRS wave complex indicating depolarization of the cardiac ventricles. The interval between the QRS wave complex and mechanical constriction is relatively fixed. Thus, the triggering signal can be used as a referenced time point for absolute phase measurement. If pulsatile pressure is assumed to be constant under normal conditions, ALDF measurement may reflect total impedance from the central cardiac to the peripheral cochlear vasculature.

By the analogy to Ohm's law, it is known that the pressure gradient along the length of an individual microvessel determines flow for a given luminal diameter and a specified magnitude of apparent blood viscosity (Lipowsky et al., 1977). The pressure gradient in a microvessel is in turn determined by the pulse pressure. Factors affecting the pulse pressure and its propagation in central vessels (such as stroke volume, heart rate, total peripheral resistance, mean circulatory filling pressure, vascular compliance) may cause change in pulsatile flow in microvessels. Thus, ALDF measurements describe the summed mechanical properties of the microvascular beds as well as the supplying vessels.

\section{Interpretation of the experimental results}

In the current experiment, the approach for evaluating ALDF as an indirect measurement of vascular mechanical status or impedance was the manipulation of cochlear circulation with photochemical damage or a vasodilator. Although the precise quantitative changes in mechanical vascular parameters such as resistance and compliance are unknown, during the manipulations significant cochlear flux changes are demonstrated by conventional LDF. Since cochlear flux is determined by the concentration and mean velocity of moving blood cells, it is a semiquantitative measurement of blood flow. According to the analogy with Ohm's law, under the condition of constant perfusion pressure, changes in steady blood flow indicate changes in resistance, while changes in pulsatile flow and in phase relationship between pulse pressure and flow indicate impedance changes. During photochemicallyinduced thrombosis of the cochlear microvasculature, ALDF reveals an illumination time-dependent decrease in both pulsatile and mean blood flow (Fig. 2 and 3). The decrease in steady blood flow is greater than that of pulse flow. In addition, the time delay of pulse flow decreases with illumination time (Fig. 4). These changes were probably caused by increased resistance, decreased capacitance, changes in interaction between blood cells and endothelial cells, and viscoelasticity of blood during photochemical damage.
The vasodilator sodium nitroprusside was applied to the round widow membrane and its effect on vascular mechanical properties was assessed with ALDF. As reported by Ohlsèn et al. (1991), application of $2 \% \mathrm{NP}$ to the round window induced a dramatic increase in $\mathrm{CBF}$, as measured with LDF. ALDF shows increases in both amplitude and time delay of pulsatile blood flow. These results suggest decreased resistance and increased capacitance in response to the vasodilating effects of NP. It is possible that nitric oxide (NO)-related interaction between platelet and endothelial cells (Graaf et al., 1992) as well as rehological changes are involved in the CBF response to topical NP applications.

\section{Predicted development and application of ALDF}

For ALDF, it is essential to have an ECG with a high signal to noise ratio, and a laser Doppler flowmeter with a short time constant and an analog signal output is recommended. An even greater improvement in repeatability would be expected when ALDF is combined with an integrated probe (multiple probe). Several signals for synchronizing flux are available: ECG (electrodes), heart sounds (microphone), pressure pulse at different locations (pressure transducer), blood flow of large supplying vessels in various locations (ultrasonic sensor or others). With different synchronizing signals, ALDF can provide differential information on vascular mechanical properties. A new generation of laser Doppler flowmeter with averaging capabilities will make ALDF even more convenient.

ALDF provides not only mean flux (related to mean blood flow) but also pulsatile flux (related to pulse blood flow) and the time delay of pulse flow. Amplitude and phase information of pulsatile flow may be used to detect primary mild changes in the microvasculature which occur in many pathologic conditions. With different synchronizing signals, ALDF can be helpful in differential diagnosis by identifying the location of vascular pathology. In the field of hearing research, ALDF may be used to study the physiology and pathology of cochlear microvasculature, such as autoregulation, sound-induced hearing loss, sudden deafness, Meniere's syndrome, aging and effects of vasoactive agents. However, the major application of ALDF is expected to be clinical in diagnosing and monitoring peripheral vascular diseases.

\section{Acknowledgments}

Authors gratefully acknowledge editorial help given by Nadine Brown and Kelly Gizowski and technical help by Robert Masta.

This work was supported by the American NIH grant DC 00105 and AG 08885, Alan Gornick Founda- 
tion and the National Natural Science Foundation of China.

\section{References}

Druce, H.M., Bonner, R.F., Patow, C., Choo, P., Summers, R.J. and Kaliner, M.A. (1984) Response of nasal blood flow to neurohormones as measured by laser-Doppler velocimetry. J. Appl. Physiol. $57,1276-1283$.

Graaf, J.C., Banga, J.E., Moncada, S., Palmer, R.M.J., Groot, P.G. and Sixma, J.J. (1992) Nitric oxide functions as an inhibitor of platelet adhesion under flow conditions. Circulation 85, 22842290.

Haberl, R.L., Heizer, M.L., Marmarou, A. and Ellis, E.F. (1989) Laser-Doppler assessment of brain microcirculation: Effect of systemic alterations. Am. J. Physiol. 256, H1247-H1254.

Hellem, S., Jacobsson, L.S., Nilsson, G.E. and Lewis, D.H. (1983) Measurement of microvascular blood flow in cancerous bone using laser Doppler flowmetry and ${ }^{133} \mathrm{X}_{\mathrm{e}}$-clearance. Int. J. Oral. Surg. 12, 165-177.

Johnson, J.M., Taylor, W.F., Shepherd, A.P. and Park, M.K. (1984) Laser-Doppler measurement of skin blood flow: comparison with plethysmography. J. Appl. Physiol. 56, 798-803.

Laurikainen, E.A., Miller, J.M., Quirk, W.S., Kallinen, J., Ren, T.Y., Nuttall, A.L., Grenman, R. and Virolainen, E. (1993a) Betahistine-induced vascular effects in the rat cochlea. Am. J. Otolarynol. 14, 24-30.

Laurikainen, E.A., Kim, D., Didier, A., Ren, T.Y., Miller, J.M., Quirk, W.S. and Nuttall, A.L. (1993b) Stellate ganglion drives sympathetic regulation of cochlear blood flow. Hear. Res. 64 . 199-204.

Lipowsky, H.H. and Zweifach, B.W. (1977) Methods for simultaneous measurement of pressure differentials and flow in single unbranched vessels of the microcirculation for rheolgical studies. Microvasc. Res. 14, 345-361.

Mcllroy, M.B, Seitz, W.S. and Targett, R.C. (1986) A transmission line model of the normal aorta and its branches. Cardiovase. Res. 20, 581-587.

Miller, J.M., Marks, N.J. and Goodwin, P.C. (1983) Laser Doppler measurements of cochlear blood flow. Hear. Res. 11, 385-394.

Miller, J.M. and Nuttall, A.L. (1990) Cochlear blood flow. In: A.P. Shepherd and P.A. Öberg (Eds.), Laser-Doppler Blood Flowmetry, Kluwer Academic Publishers, Boston, MA, pp. 319-347.

Ohlsèn, K.A., Baidwin, D.L., Nuttall, A.L. and Miller, J.M. (1991) Influence of topically applied adrenergic agents on cochlear blood flow. Circ. Res. 69, 509-518.

Quirk, W.S., Wright, J.W., Dengerink, H.A. and Miller, J.M. (1988) Angiotensin II-induced changes in cochlear blood flow and blood pressure in normotensive and spontaneously hypertensive rats Hear. Res. 33, 129-136.

Ren, T.Y., Bai, Q.S. and Li, S.Q. (1991) Effect of sympathetic stimulation on the cochlear blood flow. J. Xian Med. Univ. 12. $408-11$.

Ren, T.Y., Laurikainen, E.A., Quirk, W.S., Miller, J.M. and Nuttall, A.L. (1993a) Effects of electrical stimulation of the superior cervical ganglion on cochlear blood flow in guinea pig. Acta Otolarygol. 113, 146-151.

Ren, T.Y., Laurikainen, E.A., Quirk, W.S., Miller, J.M. and Nuttall, A.L. (1993b) Effects of stellate ganglion sfimulation on bilateral cochlear blood flow. Ann. Otol. Rhinol. Laryngol. 102, 378-384.

Ren, T.Y., Nuttall, A.L. and Miller, J.M. (1993c) Provoked flux motion of cochlear blood flow measured with laser Doppler flowmetry in guinea pig. Acta Otolaryngol. 113, 609-614.

Ren, T.Y., Nuttall, A.L. and Miller, J.M. (1993d) Contribution of the anterior inferior cerebellar artery to the cochlear blood flow in guinea pig: A model-based analysis. Hear. Res. 71, 91-97.

Riva, C.E., Petrig, B.L. and Grunwald, J.E. (1990) Retinal blood flow. In: A.P. Shepherd and P.A. Öberg, (Eds.), Laser-Doppler Blood Flownetry, Kluwer Academic Publishers, Boston, MA, pp. 349--383.

Roman, R.J. and Smits, C. (1985) Laser-Doppler determination of capillary blood flow in young and adult rats. Am. J. Physiol, 251, F115-F124

Sillman, J.S., LaRouere, M.J., Masta, R.I., Miller, J.M. and Nuttall, A.L. (1989) Electrically stimulation increases in cochlear blood flow: 1 . frequency and intensity effects. Otoläryngol. Head Neck Surg. 100, 308-316.

Shepherd, A.P. and Riedel, G.L. (1982) Continuous measurements of intestinal mucosal blood flow by laser Doppler velocimetry. Am. J. Physiol. 242, G668-G672.

Suga, F. and Snow, J.B. Jr. (1969) Cochlear blood tlow in response to vasodilating drugs and some related agents. Laryngoscope 79. 1956-1979.

Taylor, M.G. (1959) An experimental determination of the propagation of fluid oscillations in a tube with a visco-elastic wall: together with an analysis of the characteristics required in an electrical analogue Phys. Med. Biol. 4, 3-82

Tyml, K, Roman, R.J. and Lombard, J.H. (1990) Blood flow in skeletal muscle. In: A.P. Shepherd and P.̊. Öberg, (Eds.), Laser-Doppler Blood Flowmetry, Kluwer Academic Publishers, Boston, MA, pp. 215-226.

Umemura, K., Kohno, Y., Matsuno, H., Uematsu, T. and Nakashima, M. (1990) A new model for photochemically induced thrombosis in the inner ear microcirculation and the use of hearing loss as a measure for microcitculatory disorders. Eur. Arch. Otorhinolaryngol. 248, 105-108.

Womersley, J,R. (1957) Oscillatory flow in arteries: The constrained elastic tube as a model of arterial flow and pulse transmission. Phys. Med. Biol. 2, 178-187. 